\title{
Escala de Percepción de la Sexualidad en Mujeres Después del Parto
}

\section{Stephanie Fernández-Brizuela y Josiane Pawlowski}

\section{Universidad de Iberoamérica}

\section{Resumen}

La sexualidad de las mujeres se ve afectada después del parto. Por esta razón, se desarrolló una escala para evaluar la percepción de la sexualidad de las mujeres en esta etapa de la vida. El presente estudio analiza la consistencia interna y la evidencia de la validez convergente de la nueva escala en comparación con una escala de satisfacción sexual. La recolección de datos se realizó a través de una plataforma virtual. Los participantes fueron madres con hijos entre 6 meses y 3 años, que compusieron una muestra de 101 participantes entre 20 y 42 años $(\mathrm{M}=28.45, \mathrm{SD}=5.38)$. Las madres respondieron la Escala de Percepción sobre la Sexualidad en las Madres (EPSM), elaborada en el presente estudio, y la Nueva Escala de Satisfacción Sexual (NSSS). Los resultados del análisis de alfa de Cronbach fueron de .92 para la escala EPSM y de .95 para la escala NSSS. Los análisis de correlación Ítem-Total corregida y el Alfa de Cronbach si se elimina el ítem señalaron la consistencia de 32 ítems de la escala. El análisis de correlación de Pearson (-.76) fue alto entre la EPSM y la NSSS. Los resultados indican una alta consistencia interna de la escala y sugieren la exclusión de dos ítems. Además, la validez convergente se evidencia por la correlación con la escala de

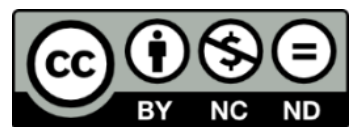


satisfacción sexual. La investigación fomenta el estudio de la percepción de la sexualidad en las madres después del parto con el objetivo de un enfoque multidisciplinario de esta población, incluida la medición con pruebas como una técnica complementaria.

Palabras clave: Percepción, sexualidad, satisfacción sexual, madres, validez 


\section{Introducción}

La sexualidad se engloba como un rasgo innato e inherente al ser humano que consta de una multiplicidad de factores, entre ellos biológicos, psicológicos, socioeconómicos, culturales, éticos, religiosos, espirituales y comunicativos (Gorguet, 2008; Rodríguez Fernández y Sueiro Domínguez, 2017). Según la Organización Mundial de la Salud (2010), la sexualidad "abarca el sexo, las identidades y los roles de género, la orientación sexual, el erotismo, el placer, la intimidad y la reproducción" (p.4). Asimismo, "se siente y se expresa a través de pensamientos, fantasías, deseos, creencias, actitudes, valores, comportamientos, prácticas, roles y relaciones" (OMS, 2010, p. 4).

En este sentido, la sexualidad no se fundamenta en la genitalidad. De otro modo, la satisfacción sexual desempeña un papel preponderante. Pérez Triviño (2013) relacionan la satisfacción sexual a las características y hábitos individuales, incluyendo la percepción de los estímulos sexuales, la capacidad de concentrarse en el estímulo sexual y sentimientos, y otras reacciones fisiológicas y psicológicas. Asimismo, para su evaluación se considera un enfoque interpersonal, el cual toma en consideración el intercambio emocional entre las parejas sexuales. Al mismo tiempo, esta podría prescindir de un enfoque dirigido a las actividades sexuales, el cual se centra en las características tales como frecuencia, variedad e intensidad. 
Tras lo anterior, es producente vislumbrar que la sexualidad no es un aspecto estático, ya que es posible que esta varíe en las distintas etapas del desarrollo de los seres humanos. Según Enderle et al. (2013), uno de los períodos más difíciles presentes en relación con la sexualidad es el período puerperal, ya que este constituye una reducción o ausentismo de actividad sexual, especialmente con relación al coito para la mayoría de las mujeres a partir de aspectos biológicos, psicológicos y sociales.

Después del parto, la mujer presenta un incremento en la prolactina y por ende una disminución de las hormonas sexuales, de modo que puede llegar a exhibir sentimientos negativos que influyen su autoestima y autoimagen circunstancialmente. En este sentido, pueden llegar a sentirse incómodas con su propio cuerpo o en la relación con su compañero o compañera sentimental y, en su sexualidad, vulnerables, cansadas en extremo, con necesidad de afecto, con miedo al nuevo desempeño sexual, con miedo al dolor y miedo a quedar de nuevo embarazadas, cambios en las rutinas, roles y responsabilidades o inclusive pueden llegar a pensar que el rol de madre no es sexy (Márquez Carrasco, 2014; Navarro-Gil y Gómez-Mariñelarena, 2016).

Asimismo, Márquez Carrasco (2014) destaca algunos aspectos físicos asociados al postparto, entre ellos:

daños en la musculatura pélvica, loquios, cambios hormonales que dan sequedad vaginal, relajación de la musculatura de introito y vagina, dificultad para compatibilizar la imagen de los pechos como fuente de alimento para el bebé y

\section{py)eon


elemento erótico, secreción láctea durante la excitación sexual y orgasmo, coitalgia y coitorragia, desgarros perineales o episiotomía (párr. 3).

De ahí que definir en la literatura un momento oportuno para reanudar las relaciones sexuales durante el puerperio sea una tarea ardua para algunos investigadores. Conforme citado por Márquez Carrasco (2014), algunos autores sugieren que el coito puede ser retomado dos semanas después de dar a luz, tomando en cuenta el sentimiento de bienestar de la mujer; mientras que otros consideran que debe ocurrir treinta días después del parto, ya que de otro modo produciría incomodidad y dificultades con el proceso curativo. De todos modos, la mayoría coincide en un rango de tiempo entre 5-12 semanas, considerando que el retorno de la actividad sexual dependerá de cuán preparada se sienta la mujer a nivel físico y psicológico.

En lo que respecta a la respuesta sexual en el postparto, González (2016) plantea un deseo sexual disminuido en mujeres en el periodo posparto. También señala que la fase de orgasmo cambia en el postparto, presentando estudios que sugieren tiempos medios distintos después del parto donde las mujeres logran la fase de orgasmo. Por ejemplo, únicamente el $20 \%$ de las mujeres experimentan un orgasmo durante la primera relación sexual tras el parto, siendo la séptima semana postparto el tiempo medio de las puérperas para el primer orgasmo tras el parto (González, 2016). Otros estudios citados por González han evidenciado que, a las 24 semanas postparto, el $61 \%$ de las mujeres logran la fase de orgasmo, y al año del nacimiento, el $77 \%$ de las mujeres describen la fase de orgasmo como similar a la previa a la 
gestación. En cuanto a la reanudación de la actividad sexual en las parejas, a la sexta-octava semana tras el parto el 40-60\% de las parejas han mantenido la primera relación coital, lo que aumenta al 80\% de las mujeres en la decimosegunda semana postparto (González, 2016).

Ahora bien, con relación a la percepción de la sexualidad, se han realizado diversos estudios y escalas enfocadas en la subjetividad del individuo con respecto a la vivencia de esta. Navarro y Gómez (2016) realizaron un estudio con una muestra de 35 mujeres puérperas, en donde se encontró que el $50 \%$ de ellas había notado una disminución en su deseo sexual y un $40 \%$ de las mujeres se percibían a sí mismas como "aún no recuperadas, pero no me veo tan mal". Acorde a lo anterior, las variables que influyeron en dichos resultados son la percepción que tienen sobre cómo creen que las ve su pareja, si creen que su pareja tiene la misma percepción que ellas tienen de sí mismas, recibir besos, abrazos y caricias de su pareja y cambios percibidos en el deseo. Esto se pudo reflejar en las declaraciones de vergüenza e incomodidad que reportaron algunas madres, las cuales evidenciaron insatisfacción con su cuerpo y una influencia negativa en su vida sexual. Concluyentemente, aquellas mujeres que se sintieron incómodas con su cuerpo en la etapa postparto tuvieron una afectación en su autoestima, autoimagen, sexualidad y la relación con su pareja.

Pérez Triviño (2013) realizó una investigación en muestra de personas usuarias de redes sociales utilizando una escala que pretendía analizar la posible relación entre diversas características relacionadas con la satisfacción sexual. La confiabilidad de los ítems en la

\section{psico


muestra evaluada fue de .93, reforzando la validez de constructo del instrumento en su versión en español, y de .90 y .93 en su versión en inglés y croata, respectivamente.

Los autores Santos et al. (2009) realizaron una investigación acerca de la fiabilidad y validez del Índice de Satisfacción Sexual (ISS) en la versión española, la cual cuenta con una fiabilidad de consistencia interna que oscila entre $\alpha=.86$ y $\alpha=.95$. El valor de consistencia interna para la escala global fue $\alpha=.89$. Además, en dicho estudio, se aplicaron una serie de escalas relacionadas con la satisfacción sexual, las cuales fueron Inventario de Deseo Sexual, Inventario de Excitación y Escala de Doble Moral. Con el Inventario de Deseo Sexual se evaluó el interés de la persona por la actividad sexual "diádica" y "solitaria”; en dicho estudio el valor alfa de Cronbach para deseo sexual diádico fue $\alpha=.89$ y $\alpha=.91$ para deseo sexual solitario. Por su parte, el Inventario de Excitación evaluó el grado de excitación experimentado ante diferentes actividades sexuales, con el cual se logró un valor de fiabilidad de consistencia interna de $\alpha=.90$. Finalmente, con la Escala de Doble Moral se evaluó la doble moral en el área de la sexualidad, donde se obtuvo un valor alfa de Cronbach de $\alpha=$. 79. Cabe resaltar que, en esta última, Caron et al. (1993) informaron de una consistencia interna igual a $\alpha=.72$; así como Sierra et al. (2007) hallaron valores iguales a $\alpha=.70$ y $\alpha=$ .76 para mujeres y hombres, respectivamente (Santos et al., 2009).

En el caso de Blümel et al. (2004), se realizó una investigación en torno a la evaluación de la sexualidad femenina en Chile. Su objetivo consistió en aplicar y validar el Índice de Función Sexual Femenina en población chilena, establecido en el International 
Consensus Development Conference on Female Sexual Dysfunctions en mujeres de 20 a 59 años con actividad sexual. Entre sus variables tomaba en cuenta deseo, excitación, lubricación, orgasmo, satisfacción y dolor. Como resultados de este índice se obtuvo una consistencia interna mayor de .70 .

Por su parte, Soria (2012) estudiaron la Escala de Valoración de la Actividad Sexual EVAS-M, que consta de 8 reactivos para valorar la satisfacción, el deseo, la excitación, la lubricación, el orgasmo, el dolor y la cercanía emocional y confianza con la pareja para la valoración de la actividad sexual de la mujer. La función de esta escala es discriminar las áreas problemáticas de la actividad sexual proporcionando una herramienta para el cribado de la disfunción.

Otro hallazgo por destacar es el de Paredes y Pinto (2009), que encontraron una relación entre la imagen corporal y la satisfacción sexual al evaluar una muestra de jóvenes mujeres universitarias. Los autores aplicaron la prueba Body Shape Questionnaire y el Inventario de Satisfacción Sexual. En la muestra evaluada, se encontró un nivel de alfa de Cronbach de .897 para el Inventario de Satisfacción Sexual y de .914 para los ítems el instrumento Body Shape Questionnaire, sugiriendo la consistencia interna de los ítems de cada una de las pruebas.

Así, es un hecho que el período puerperal está mediado por una multiplicidad de factores, de modo que se enmarca como un fenómeno importante a estudiar. Parte de las razones subyacentes que motivan el presente estudio se derivan de la ausencia de

\section{psico


investigaciones que versen en torno a la temática seleccionada, más aún de la ausencia de una escala estandarizada en Costa Rica para medir dicho constructo. Con la escala se busca establecer la percepción sobre la sexualidad en mujeres madres, de modo que sea posible identificar aquellas áreas más afectadas con el fin de que, posteriormente, sea posible ofrecer una mejor calidad de vida sexual bajo una modalidad multidisciplinaria, preferiblemente. Así, el objetivo de la presente investigación consiste en desarrollar una escala que evalúe la percepción con respecto a la sexualidad en madres con hijos en edades entre los 6 meses y 3 años de manera válida y confiable.

\section{Método}

\section{Participantes}

La muestra se conformó por 101 participantes en un rango de edad de 20 a 42 años $(\mathrm{M}=28.45 ; \mathrm{DS}=5.38)$, todas madres con hijos en edades entre los 6 meses y los 3 años. De estas, 98 fueron costarricenses (97\%) y 3 fueron extranjeras: 1 de nacionalidad española (1\%), 1 de nacionalidad colombiana (1\%) y 1 de nacionalidad nicaragüense (1\%). La mayor parte de las participantes son de la Gran Área Metropolitana (GAM), un 46.5\% de San José, 29.7\% de Heredia, $10.9 \%$ de Cartago, $8.9 \%$ de Alajuela y 4\% de Puntarenas. Otras características demográficas de la muestra se especifican en la Tabla 1.

Respecto a algunas características del parto, 68 de las participantes tuvieron un parto a término $(67,3 \%)$, mientras que 26 , un parto inducido $(25,7 \%)$ y 7 un parto prematuro 
$(6,9 \%)$. Respecto a las complicaciones durante el proceso de parto, 30,7\% de la muestra señaló lo siguiente: a 25 de las participantes les realizaron una episiotomía $(24,8 \%), 22$ de ellas tuvieron desgarros durante el parto $(21,8 \%)$ y a 3 de las participantes se utilizó el fórceps (3\%). En lo que atañe a posibles complicaciones con el bebé durante el parto, 13 de las participantes $(12,9 \%$ de la muestra total), registraron algún tipo de complicación con el bebé. Asimismo, se les preguntó a las madres por las complicaciones después del parto, respecto al que se encuentra: depresión postparto $(18,8 \%)$, estreñimiento $(16,8 \%)$, hemorroides $(11,9 \%)$, mastitis $(9,9 \%)$, infecciones de la cesárea (2\%), infecciones de episiotomía (2\%), incontinencia urinaria (2\%) y hemorragias $(2 \%)$.

Tabla 1. Características de la muestra

$n$

Escolaridad

Primaria

Secundaria

Superior

Posgrado

No indicado
3

\section{py)


Estado civil

$\begin{array}{lcc}\text { Casada } & 44 & 43.6 \\ \text { Unión Libre } & 28 & 27.7 \\ \text { Soltera } & 27 & 26.7 \\ \text { Divorciada } & 2 & 2\end{array}$

Cantidad de hijos(as)

1

2

3

5

Tipo de parto

Vaginal

Cesárea
69

26

4

2
68.3

4

2

58

57,4

43

42,6

\section{Instrumentos}

Escala Percepción sobre la Sexualidad en Madres (EPSM): Construida en el curso y principal variable del presente estudio. El instrumento busca evaluar la percepción con 
respecto a la sexualidad en madres con hijos en edades entre los 6 meses y 3 años, y se compone de 34 ítems que puntúan en una escala tipo Likert de 5 categorías desde 1 "muy en desacuerdo" hasta 5 "muy de acuerdo".

Nueva Escala de Satisfacción Sexual (NSSS), desarrollada por Pérez Triviño (2013): Consiste en una escala de 20 reactivos estilo Likert de 5 categorías, desde 1 "nada satisfecho" hasta 5 "extremadamente satisfecho". Un estudio con una muestra española indicó una confiabilidad de .93 evaluada por el Alfa de Cronbach. Su validez se estableció a partir de la validez de constructo del instrumento en su versión en español.

\section{Procedimiento}

El presente estudio es de tipo cuantitativo y correlacional (Hernández, Fernández, y Baptista, 2010). La construcción de la escala EPSM fue elaborada con la colaboración de graduados en Bachillerato en Psicología, estudiantes del grado de Licenciatura en Psicología del segundo cuatrimestre del año 2018 de la Universidad de Iberoamérica de Costa Rica, bajo la supervisión de la profesora del curso. En primera instancia se definió el constructo y sus dimensiones; posteriormente se crearon los ítems en conjunto como grupo.

Para la construcción de la escala se tomó en cuenta la opinión de cuatro expertos que han trabajado con la población que se pretende evaluar: profesionales en medicina general, ginecología, psicología y sexología. Los expertos revisaran la escala y agregaron sugerencias para adecuar los reactivos al contenido teórico. Además, se utilizó el método de Entrevista

\section{psico


Cognitiva (Smith-Castro y Molina, 2011) que fue aplicada a diez participantes, de modo que con esta pudieran añadirse nuevas correcciones. Los resultados permitieron mejorar la comprensión de los ítems y su construcción gramatical. La escala resultó con 34 reactivos, siendo 23 negativos y 11 positivos. Para investigar la consistencia interna de la escala, se invirtieron los resultados de los ítems positivos, dada la naturaleza de los reactivos, por lo que el resultado obtenido en la escala pasó a reflejar características negativas relacionadas a la percepción sobre la sexualidad. Los ítems positivos eran el 1, 2, 8, 9, 13, 16, 20, 21, 23, 24 y 27, cuya descripción se puede encontrar en la Tabla 2.

La evaluación de los participantes fue voluntaria, sin ninguna compensación por su contribución al estudio. Se utilizó un muestreo no probabilístico por conveniencia. El criterio de inclusión fue ser madre de un hijo en edades entre los 6 meses y 3 años y el de exclusión, madres que solamente tenían hijos con más de 3 años. La recolección de datos fue virtual; se les envió una invitación a las madres con un enlace para una encuesta que contenía un consentimiento informado, preguntas relacionadas a datos personales y sobre el parto, la escala desarrollada en esta investigación, así como la escala NSSS. La investigación se realizó bajo las disposiciones señaladas en el Código de Ética del Colegio de Profesionales en Psicología de Costa Rica.

\section{Análisis de datos}


Todos los análisis estadísticos se llevaron a cabo con el Programa Estadístico SPSS, versión 23 (SPSS Inc., Chicago, Illinois, USA). Se realizó el análisis descriptivo de media y desviación estándar de la variable edad, y se hizo el análisis descriptivo de frecuencias para las variables provincia de residencia, escolaridad, estado civil, cantidad de hijos, tipo de parto y complicaciones e intervenciones en el parto. Se utilizó el alfa de Cronbach para medir el nivel de confiabilidad de la prueba. Además, se aplicó la correlación ítem-total corregida y el Alpha de Cronbach para la escala si se elimina el ítem. También se aplicó la prueba de normalidad de Kolmogrov-Smirnov para verificar la distribución de las variables de suma total de cada escala evaluada, así como se realizó el análisis de asimetría y curtosis para contrastar dicha prueba de normalidad, tomando como referencia la recomendación de distribuciones con coeficientes de asimetría y curtosis en el rango $(-1,1)$ (Ferrando y Anguiano-Carrasco, 2010; Muthén y Kaplan, 1985, 1992). Por último, se utilizó la correlación de Pearson para evaluar la relación entre las dos escalas.

\section{Resultados}

Dados los aportes de los participantes en la entrevista cognitiva se pudo identificar que la primera versión de la escala presentaba instrucciones con una consigna muy grande y que algunos ítems eran difíciles de comprender. Como resultad, la instrucción fue disminuida para mejorar la comprensión y se realizó una revisión de la redacción de los ítems señalados. Al mismo tiempo, los expertos indicaron que ciertos ítems no tenían relevancia para el

\section{psico


propósito de la investigación, lo cual ayudó a orientar lo que se le estaba preguntando a los participantes con la finalidad de tener un propósito más claro y completo. Las sugerencias permitieron modificar la redacción y contenido de los ítems. Posteriormente, se realizó una prueba piloto con la cual se pudo determinar que la comprensión de los ítems era adecuada.

La confiabilidad fue determinada por medio de la consistencia interna de reactivos, obteniéndose un coeficiente de alfa de Cronbach de .92 para la escala EPSM y de .95 para la escala NSSS. La consistencia interna de la prueba ESPM fue verificada con la correlación ítem-total corregida y el Alpha de Cronbach para la escala si se elimina el ítem, cuyos resultados pueden ser observados en la Tabla 2. Los resultados indican dos ítems (ítem 9 y ítem 16) con correlaciones negativas y que si eliminados mejoran la confiabilidad de la escala.

Tabla 2. Correlación Ítem-Total Corregida y Alpha de Cronbach si se elimina el Ítem

\begin{tabular}{ccc}
\hline Ítem Descripción del ítem & $\begin{array}{c}\text { Correlación } \\
\text { Ítem-Total } \\
\text { Corregida }\end{array}$ & $\begin{array}{c}\text { Alpha de } \\
\text { Cronbach si se } \\
\text { elimina el Ítem }\end{array}$ \\
\hline
\end{tabular}


1 Considero que mi vida sexual es muy excitante y satisfactoria.

2 Siento la misma atracción sexual por mi pareja después del nacimiento de mi bebé.

3 Después del nacimiento del bebé, siento inseguridad al mostrar mi cuerpo durante la relación sexual. parto prolongó el tiempo para retomar mi vida sexual.

$5 \quad$ Siento culpa de que mi vida sexual no sea satisfactoria desde que nació el bebé.

6 Tengo miedo a sentir dolor durante el acto sexual desde que nació mi bebé.

$7 \quad$ Necesito asegurarme de que todas las tareas de cuido del bebé se hayan realizado para poder concentrarme durante el coito.

8 Después del nacimiento del bebé, me siento dispuesta a satisfacer las necesidades sexuales de mi pareja igual que antes.

9 He percibido cambios en mi apariencia física después del nacimiento de mi bebé que contribuyeron a mi vida sexual.

10 Pienso que ha disminuido mi deseo sexual desde el nacimiento de mi bebé.

11 Después del nacimiento del bebé, tengo relaciones sexuales solo para complacer a mi pareja.

12 Creo que la satisfacción sexual se ha reducido desde el nacimiento de mi bebé.

13 Logro hablar abiertamente con mi pareja sobre mis necesidades sexuales.

14 Mantener relaciones sexuales no es una prioridad desde el nacimiento de mi bebé. 
15 Me reprocho por no tener tiempo de intimidad con mi pareja.

16 La experiencia del parto ha afectado positivamente en mi motivación sexual.

17 Pienso que los cambios físicos después del nacimiento de mi bebé perjudicaron mi vida sexual.

18 Después del nacimiento del bebé, los sentimientos de rechazo hacia mi cuerpo han afectado mi sexualidad.

19 Desde el nacimiento de mi bebé tengo un sentimiento de culpa después de tener relaciones sexuales.

20 Conocer los cambios que suceden en mi cuerpo después del parto mejora las relaciones sexuales.

21 Después del nacimiento del bebé, me importan las necesidades sexuales de mi

22 La lactancia materna genera cambios negativos en la actitud de mi pareja hacia la sexualidad.

23 Las relaciones sexuales siguen igual de espontáneas como antes del nacimiento del bebé.

24 Soy capaz de alcanzar el clímax sexual (orgasmo) con la misma frecuencia que antes del nacimiento del bebé.

25 Se necesita planificar el momento para tener relaciones sexuales después del nacimiento del bebé.

26 El cambio en mi horario de sueño podría afectar en mi sexualidad.

27 Me excito con facilidad aún después del nacimiento del bebé.

28 Mis prioridades han cambiado entorno al bebé y he olvidado tener tiempo para mí misma. 
29 Considero que la frecuencia con la que tengo relaciones sexuales ha disminuido desde el nacimiento de mi bebé.

30 Preocuparme de tener otro embarazo disminuye mi deseo sexual.

31 Después del nacimiento del bebé, he disminuido mi actividad sexual debido al dolor que siento en el acto.

32 Pienso que si tengo relaciones sexuales con mi pareja descuido las atenciones que debo darle a mi bebé.

33 La forma en que ocurrió el parto (vaginal o cesárea) ha afectado negativamente mi vida sexual.

34 Me culpo por no satisfacer las necesidades sexuales de mi pareja.

En lo que respecta a la prueba de normalidad de Kolmogorov-Smirnov aplicado para las variables de suma total de cada escala, esta resultó no significativo para la Suma EPSM $(p=.17)$, no obstante, en la Suma NSSS resultó significativa $(p=.01)$; esto puede derivarse por el tamaño de la muestra o número de ítems. Según los análisis obtenidos de asimetría $($ Suma ESPM= .18; Suma NSSS= .06) y curtosis $($ Suma ESPM= .-68; Suma NSSS= -.63), se considera que los resultados aún se acercan a la normalidad. Para la evaluación de la validez convergente se realizó la correlación de Pearson entre la escala ESPM y NSSS, obteniéndose -.76. Esto indica que la relación entre ambas escalas es alta y muestra que entre más negativa

\section{PSteo-


es la percepción sobre la sexualidad, menor es la satisfacción sexual que presentan las mujeres después del parto.

\section{Discusión}

El presente estudio buscó crear una escala que midiera la percepción de la sexualidad en madres en período postparto en una muestra de mujeres con hijos en edades entre los 6 meses y 3 años. De los datos obtenidos, la confiabilidad de la escala (.92) resultó como excelente, considerando como valor mínimo el .8 (Cea, 1999). Con respecto a la escala NSSS, el resultado fue de .96 , evidenciando un aumento de fiabilidad de la escala en la muestra contemplada para este estudio cuando comparada a la muestra española con la que se obtuvo un alfa de Cronbach de .93 (Pérez Triviño, 2013). Esto indica que ambas escalas son consistentes en esta muestra ya que se obtuvo un coeficiente de alfa excelente (> .90) para ambas escalas (George y Mallery, 2003).

En cuanto a la consistencia interna, de los 34 ítems creados para la ESPM, 32 reactivos se presentan homogéneos y contribuyen para la medida del constructo evaluado. Entre los ítems que mostraron bajo nivel de correlación y que, se excluidos, permiten que la escala aumente su homogeneidad, están el 9 y el 16, los cuales se relacionan con los cambios físicos de la mujer y la influencia positiva de los mismos en su vida sexual. La baja correlación del ítem 9 al total, que indagaba sobre si los cambios en la apariencia física después del nacimiento del bebé contribuyeron a la vida sexual, indica la posibilidad de que 
las mujeres entrevistadas no percibieran que los cambios sufridos tras su embarazo y posterior al parto se relacionaran de una u otra manera dentro del ámbito de su vida sexual (Rejane, Matos y Rosa, 2010). Según Álvarez, Gutierréz y Velásquez (2016), es común que la autoestima de la mujer juegue un papel importante en la percepción de sí misma, y en todos los cambios físicos que se sufren a lo largo de este proceso, por lo que puede verse interferida de una manera considerable la motivación o el impulso a la hora de mantener actos sexuales. De igual manera, comentan que en ocasiones el aparato reproductivo femenino al no estar completamente recuperado puede generar desmotivación. Así mismo, en el ítem 16 que pretendía reflejar si la experiencia del parto ha afectado positivamente en la motivación sexual también no contribuye a la homogeneidad de la escala por razones similares. Tras lo anterior, dichos ítems no contribuyen a la consistencia interna de la escala, por lo que podrían ser eliminados.

Otros ítems de la escala que fueron planteados para ser positivos y relacionados a una mejora de la percepción sobre la sexualidad podrían ser replanteados, tal como el ítem 20, el cual pretendía evaluar si conocer los cambios que suceden en el cuerpo femenino posterior al parto podrían mejorar las relaciones sexuales. Es importante señalar también que, según Ortega (2013), las mujeres se enfocan en múltiples ocasiones en buscar satisfacer un ideal propuesto por la sociedad, lo que genera en esta etapa que la mujer busque como satisfacer su necesidad de aprobación por sí misma y la aprobación por parte de su pareja en otros recursos fuera del ámbito sexual.

\section{psico


En cuanto a la validez, el resultado de correlación moderado entre la escala desarrollada EPSM y la escala NSSS indican evidencias de validez convergente. Al ser un resultado negativo, señalase que la escala ESPM es dirigida a evaluar percepciones negativas sobre la sexualidad en la etapa postparto, y que, cuanto más negativa es esta percepción, menor es la satisfacción sexual de las mujeres. La NSSS fue elegida para la comparación con la ESPM porque como tal no existe una escala que se centre en evaluar las percepciones sobre la sexualidad en el período postparto, por lo que se contrastó con una que midiera la satisfacción sexual. Además, la escala NSSS es corta, reportó una buena confiabilidad en los estudios originales en inglés y con una muestra española, la cual se confirma con la muestra en su mayoría costarricense contemplada en este estudio. La escala NSSS tiene un enfoque multidimensional, buscando distinguir entre evaluación de la satisfacción con la relación y la satisfacción sexual personal y la de cada miembro de la pareja (Pérez Triviño, 2013).

Entre las limitaciones del presente estudio, se debe considerar la muestra, aunque adecuada a los objetivos de la investigación, considerando las características de inclusión que debían existir, podría ser mayor en investigaciones futuras con la finalidad de obtener datos más representativos. Además, la recolección virtual, aunque sugiere Pérez Triviño (2013) que "las encuestas online proporcionan algunos beneficios (por ej. mejora el anonimato y la confidencialidad) incluso más que en las formas tradicionales de recolección de datos” (p. 34), hay que considerar, tal como la misma autora señala que, "existe menor control sobre las condiciones durante la participación, pudiendo aumentar la falsificación, los datos incompletos y el sesgo de autoselección" (p. 34). La encuesta online puede representar un 
obstáculo para ciertas participantes, ya que algunas mujeres solamente contestaron la primera parte de los reactivos, correspondientes a la ESPM, además de la necesidad de exclusión de participantes que no cumplían con los criterios de inclusión, por ejemplo, mujeres con hijos de más de 3 años que contestaron a toda la encuesta.

Para futuros estudios, se sugiere la adecuación de los ítems de la escala siguiendo procedimientos más rigurosos. De todos modos, el presente estudio constituye un primer acercamiento al fenómeno en Costa Rica, lo que registra su importancia, de modo que podría ser considerado como una base exploratoria para futuros estudios o inclusive ser replicado con características representativas de la muestra, como la edad de la madre y del bebé o por ubicación sociodemográfica. También se sugiere tomar en consideración madres extranjeras para un posible estudio correlacional, de modo que sea posible verificar si la percepción sobre la sexualidad que se ha evidenciado en este estudio es propiamente de la percepción de las madres costarricenses.

\section{Conclusión}

Esta investigación buscó desarrollar una escala para conocer la percepción de las madres sobre la sexualidad en la etapa postparto y conocer si esta etapa tuvo alguna afectación positiva o negativa en su sexualidad. La validez de contenido de la escala se obtuvo mediante la evaluación por expertos y entrevista cognitiva, la confiabilidad por el análisis de la consistencia interna y la validez de constructo mediante evidencia de

\section{psico


homogeneidad y evidencia de validez convergente. Se puede concluir que, si se perfecciona un poco más los ítems de la escala y se aumenta la muestra, se puede obtener resultados más sólidos de la percepción de las mujeres en etapa postparto, sin embargo, esta escala mostró indicaciones de un instrumento fiable para medir la percepción de la sexualidad.

A pesar de las limitaciones presentadas en este estudio, los resultados obtenidos reflejan relevancia para el contexto costarricense, dado a que la sexualidad en el período postparto es un tema con poco auge a nivel nacional. La escala desarrollada resulta el primer instrumento, hasta donde se conoce, en el país para edificar una línea de conocimiento y aplicación en torno al constructo elegido.

\section{Referencias}

Álvarez, R., Gutiérrez, Y., y Velásquez, D. (2016). Vivencias de la sexualidad en el embarazo. (Tesis de Bachillerato). Pontificia Universidad Javeriana, Bogotá. Recuperado

de https://repository.javeriana.edu.co/bitstream/handle/10554/20445/AlvarezLeanoRut hKatherine2016.pdf? sequence $=1$

Blümel, J. E., Binfa, L., Cataldo, P., Carrasco, A., Izaguirre, H., y Sarrá, S. (2004). Índice de función sexual femenina: un test para evaluar la sexualidad de la mujer. Revista chilena de obstetricia y ginecología, 69(2), 118-125. doi: 10.4067/S071775262004000200006 
Cea, M. A. (1999). Metodología cuantitativa: estrategias y técnicas de investigación social. Madrid, España: Síntesis.

Enderle, C., Pereira, N., Lerch, V., Magroski, C., Mattos, L., y Fonseca, E. (2013). Condicionantes y/o determinantes del retorno a la actividad sexual en el puerperio. Revista Latino-Americana de Enfermagem, 21(3), 01-07. Recuperado de: http://www.scielo.br/pdf/rlae/v21n3/es_0104-1169-rlae-21-03-0719.pdf

Ferrando, P. J., y Anguiano-Carrasco, C. (2010). El análisis factorial como técnica de investigación en psicología. Papeles del Psicólogo, 31(1), 18-33. Recuperado de http://www.redalyc.org/articulo.oa?id=77812441003

George, D., \& Mallery, P. (2003). SPSS for Windows step by step: A Simple Guide and Reference. 11.0 Update (4. ${ }^{\mathrm{a}}$ ed.). Boston, USA: Allyn \& Bacon

González, L. (2016). La sexualidad femenina en el postparto: Una investigación cualitativa desde la teoría fundamentada (Tesis de Maestría). Recuperado de http://repositorio.unican.es:8080/xmlui/bitstream/handle/10902/8559/GonzalezRobl esL.pdf? sequence $=1 \&$ is Allowed $=\mathrm{y}$

Gorguet, I. (2008). Comportamiento Sexual Humano. Universidad de Ciencias Médicas de Santiago de Cuba. Recuperado de: http://tesis.repo.sld.cu/124/1//liana_Gorguet_PiLIBRO_.pdf

\section{psico


Hernández Sampieri, R., Fernández Collado, C., y Baptista Lucio, M. (2010). Metodología de la Investigación. Quinta Edición. México D.F., México: McGrawHill.

Márquez Carrasco, A. M. (2014). Dificultades en el reinicio de las relaciones sexuales de la pareja en el período puerperal. Biblioteca Las Casas, 10(3). Recuperado de http://www.index-f.com/lascasas/documentos/lc0790.pdf

Muthén, B., \& Kaplan, D. (1985). A Comparison of Some Methodologies for the Factor Analysis of Non-Normal Likert Variables. British Journal of Mathematical and Statistical Psychology, 38(2), 171-189. doi: 10.1111/j.2044-8317.1985.tb00832.x

Muthén, B., \& Kaplan, D. (1992). A Comparison of Some Methodologies for the Factor Analysis of Non-Normal Likert Variables: A note on the size of the model. British Journal of Mathematical and Statistical Psychology, 45, 19-30. doi: 10.1111/j.20448317.1992.tb00975.x

Navarro-Gil, C., y Gómez-Mariñelarena, C. (2016). Autoestima y autoimagen de las mujeres en el postparto. Medicina Naturalista, 10(1), 28-33. Recuperado de https://dialnet.unirioja.es/descarga/articulo/5302216.pdf

World Health Organization. (2010). Developing sexual health programmes: a framework for action. World Health Organization. Recuperado de http://www.who.int/iris/handle/10665/70501 
Ortega, A. (2013). Antes, durante y después del embarazo adolescente: Proyectos de vida y vivencias sobre su sexualidad. (Tesis de maestría). Recuperado de: http://bdigital.unal.edu.co/11724/1/489185.2013.pdf

Paredes, J., \& Pinto, B. (2009). Imagen corporal y satisfacción sexual. Ajayu, 7(1), 28-47. Recuperado de http://www.redalyc.org/articulo.oa?id=461545467002

Pérez Triviño, F. (2013). Nueva escala de satisfacción sexual (NSSS) en usuarios de redes sociales (Trabajo Fin de Máster). Universidad de Almería, España. Recuperado de http://repositorio.ual.es/bitstream/handle/10835/2366/Trabajo.pdf?sequence=1\&isA llowed=y

Rejane, N., Matos, N,. Rosa, D. (2010). Cuerpo y sexualidad: experiencias de puérperas. Revista Latino Americana de Enfermagem, 18 (4). 367-543. Recuperado de: http://www.scielo.br/pdf/rlae/v18n4/es_11.pdf

Rodríguez Fernández, B., \& Sueiro Domínguez, E. (2017). Sexualidad en embarazo y postparto: la necesidad de educación afectivo-sexual. Revista de Estudios $e$ Investigación en Psicología y Educación, (14), 245-248. https://doi.org/10.17979/reipe.2017.0.14.3034

Santos Iglesias, P., Sierra, J. C., García, M., Martínez, A., Sánchez, A., \& Tapia, M. I. (2009). Índice de Satisfacción Sexual (ISS): un estudio sobre su fiabilidad y validez. International Journal of Psychology and Psychological Therapy, 9(2), 259-273. Recuperado de http://www.redalyc.org/articulo.oa?id=56012878008

\section{py $\int_{\text {Lom }}$


Smith-Castro, V., \& Molina, M. (2011). La entrevista cognitiva: Guía para su aplicación en la evaluación y mejoramiento de instrumentos de papel y lápiz. Serie Cuadernos Metodológicos. San José, Costa Rica: Instituto de Investigaciones Psicológicas.

Soria, B. (2012). Estudio de la Actividad Sexual de la Mujer en el Embarazo y el Puerperio. (Tesis de maestría). Recuperado de: http://repositorio.ual.es/bitstream/handle/10835/2543/Trabajo.pdf?sequence

Agradecimientos: Las autoras agradecen al grupo de estudiantes del Curso de Seminario Avanzado en Investigación Psicológica (segundo cuatrimestre del año 2018) del grado de Licenciatura en Psicología de la Universidad de Iberoamérica de Costa Rica por la colaboración en la construcción de los ítems y en la recolección de los datos para composición de la muestra. 